\title{
Fabrication of Tamarindus indica seeds extract loaded-cream for photo-aged skin: Visioscan ${ }^{\circledR}$ studies
}

\author{
Muhammad Khurram Waqas ${ }^{1}$, Barkat Ali Khan², Naveed Akhtar ${ }^{3}$, Farzana Chowdhry', Haroon Khan², Sattar Bakhsh², \\ Shahzeb Khan ${ }^{4}$, Akhtar Rasul ${ }^{5}$
}

${ }^{1}$ Institute of Pharmaceutical Sciences, University of Veterinary and Animal Sciences, Lahore, Pakistan ${ }^{2}$ Faculty of Pharmacy, Gomal University D.I Khan, Dera Ismail Khan, Pakistan

${ }^{3}$ Department of Pharmacy, Faculty of Pharmacy and Alternative Medicine, The Islamia University of Bahawalpur, Bahawalpur, Pakistan ${ }^{4}$ Department of Pharmacy, University of Malakand, Chakdara, Pakistan

${ }^{5}$ Faculty of Pharmaceutical Sciences, Government College University, Faisalabad, Pakistan

Adv Dermatol Allergol 2017; XXXIV (4): 339-345

DOI: https://doi.org/10.5114/ada.2017.69314

\begin{abstract}
Introduction: Intracellular and extracellular oxidative stress triggered by free radicals promotes skin aging, which is designated by atypical pigmentation and wrinkles. The consumption of antioxidants is an efficacious measure to avert symptoms involved in skin aging.

Aim: The current research was commenced to explore the anti-aging potential of antioxidants present in Tamarindus indica seeds extract.

Material and methods: Tamarindus indica seeds extract was obtained by concentrating the ethanolic extract of seeds. The antioxidant activities of the extract were measured by nitric oxide radical scavenging assay, 2,2-diphenyl-1-picrylhydrazyl (DPPH) radical scavenging assay, hydroxyl radical scavenging assay and superoxide radical scavenging assay. Formulation comprising $4 \%$ of the concentrated extract of seeds was formulated by loading it in the internal aqueous phase of water-in-oil (W/O) cosmetic emulsion. The base, used as control, consisted of the same emulsion but without loading Tamarindus indica seeds extract. The cosmetic emulsions were applied to the cheeks of 11 healthy male volunteers for duration of 12 weeks. Both base and formulation were assessed for their antioxidant effects on different skin parameters i.e. skin moisture contents, elasticity and surface evaluation of living skin (SELS).

Results: The formulation showed statistically significant $(p \leq 0.05)$ and the base showed insignificant $(p>0.05)$ effects on skin elasticity and skin moisture contents. There is a significant decline in SELS, skin scaliness (SEsc), skin wrinkles (SEw), skin smoothness (SEsm), and skin roughness (SEr) parameters after application of the formulation. Conclusions: Topical application of the cosmetic emulsion entrapped with Tamarindus indica seeds extract containing various antioxidants exerts potential skin antiaging effects.
\end{abstract}

Key words: Tamarindus indica, skin elasticity, surface evaluation of living skin.

\section{Introduction}

Ultraviolet (UV) radiation from sun triggers free radical generation leading to visible symptoms of skin aging. The traditional treatment regimens comprise profoundly natural products to have fresh, supple, and younger-looking skin [1]. Antioxidant supplements are very important to maintain mechanics of human skin. Over the past decade, there has been fervent interest in products found in nature because of their perceived safety. Oxidative stress initiated by reactive oxygen species (ROS) genera- tion is an important factor modulating skin alterations, especially those caused by UV exposure and aging. The human body has several endogenous oxidative stresseliminating systems. Treatment with some antioxidants, such as ascorbic acid, tocopherols, and polyphenols, should be effective to enhance resistance to oxidative stress and prevent/improve skin aging [2].

Skin care products are often developed from plants. Many believe that if a product can be safely ingested, it will also be safe for topical application. In general, plant-

Address for correspondence: Barkat Ali Khan PhD, Faculty of Pharmacy, The Islamia University of Bahawalpur, 63100 Bahawalpur, Pakistan, phone: +92 3216808955, fax: +92 629255243, e-mail: barki.gold@gmail.com

Received: 14.05.2016, accepted: 11.06.2016. 
derived, botanical cosmeceutical products tend to be antioxidant in action since these organisms must thrive in constant direct ultraviolet (UV) light, the Earth is the most prolific manufacturer of free radicals [3].

Several classes of emulsion may be distinguished, namely oil-in-water (O/W), water-in-oil (W/O) and oil-inoil (O/O). Water-in-oil emulsion consists of water phase, which is an internal/dispersed phase, mixed with oil, which is a continuous phase. This emulsion type is often more difficult to prepare and stabilize since it is most often based on total non-emulsifiers. However recent advances in silicon chemistry and polymer chemistry have allowed preparation of excellent water-in-oil (W/O) emulsions. A real benefit of these vehicle emulsions is that they are readily spread on to the lipophilic skin and provide a film which is resistant to water wash off. This is how water resistant moisturizing cosmeceuticals are created [4].

Tamarindus indica $L$. seeds are important sources of antioxidant activity as 2-hydroxy-3',4-dihydroxyacetophenone, metdihydroxybenzoate, 3,4-dihydroxyphenylacetate and (-)-epicatechin, in addition to oligomeric proanthocyanidins (OPC). The OPCs are potent antioxidant, anti-inflammatory, antihistaminic agents and UV protective compounds. The OPCs also stabilize elastin, collagen and ground substances. The detailed study of the composition of Tamarindus indica $L$. seeds will bring contribution to health professionals not only regarding its composition but also to evaluate its antioxidant potential, fatty acid profile and content of tocopherols. Biological activity assessment of Tamarind seed was reported on the radical scavenging, lipid peroxidation reducing and anti-microbial activities including anti-inflammatory potential [5]. These findings led to the interest in using the extract derived from the seeds of tamarind for cosmetics.

\section{Aim}

The purpose of the present work is to develop a stable formulation of W/O cosmetic emulsion followed by inclusion of Tamarindus indica seeds extract containing various botanical antioxidants and its effects were analyzed on different skin related parameters by applying using non-invasive bio-physical techniques.

\section{Material and methods}

\section{Materials}

Tamarindus indica seeds were obtained from a local market of Bahawalpur, Pakistan and authenticated by the CIDS (Cholistan Institute of Desert Plants Studies), The Islamia University of Bahawalpur, Pakistan. For future reference, a voucher specimen (Voucher no. TI-SD-6-15-87) has been kept in the herbarium at CIDS. Abil EM $90^{\oplus}$ was purchased from the Franken Chemicals and paraffin oil was purchased from Merk KGaA Darm- stadt (Germany). Ethanol was taken from BDH England. Distilled water was prepared in the Cosmetics Laboratory, Department of Pharmacy, The Islamia University of Bahawalpur, Pakistan.

\section{Plant material and preparation of the extract}

The air dried and crushed seeds were extracted with ethyl alcohol for $30 \mathrm{~min}$, at a $1: 3$ seed : ethyl alcohol ratio, under continuous agitation at room temperature. Then the mixture was filtered and subjected to rotary evaporation under pressure reduced to $40^{\circ} \mathrm{C}$.

\section{Antioxidant activity of extract \\ $D P P H$ free radical scavenging activity}

Stock solution of DPPH (33 mg/l) was prepared in methanol, which gave initial absorbance of 0.493 . Stock solution $(5 \mathrm{ml})$ was added to $1 \mathrm{ml}$ of extract solution at different dilutions $(250-1500 \mathrm{\mu g} / \mathrm{ml})$. After $30 \mathrm{~min}, \mathrm{ab}-$ sorbance was measured at $517 \mathrm{~nm}$ spectrophotometrically and concentration was calculated from the standard calibration curve. Scavenging activity was expressed as the percentage inhibition calculated using the following formula:

$$
\text { Inhibition }(\%)=\frac{A_{0}-A_{1}}{A_{0}} \times 100,
$$

where $A_{0}=$ absorbance of the control and $A_{1}=$ absorbance of the extract. Scavenging activity was compared with ascorbic acid.

\section{Nitric oxide scavenging assay}

Nitric oxide scavenging activity was measured spectrophotometrically by mixing sodium nitroprusside ( $5 \mathrm{mM}$ ) dissolved in phosphate buffered saline with different concentrations of methanolic extract (250-1500 $\mu \mathrm{g} /$ $\mathrm{ml}$ ). After ambient incubation for $30 \mathrm{~min}, 1.5 \mathrm{ml}$ of this solution was taken and diluted with $1.5 \mathrm{ml}$ of Griess reagent (prepared by dissolving 1\% sulphanilamide, 2\% phosphoric acid and 0.1\% N-1-naphthylethylenediamine dihydrochloride). During reaction, diazotization of nitrite with sulphanilamide and subsequent coupling with $\mathrm{N}$-1-naphthylethylene diamine dihydrochloride resulted in the formation of active chromophore that was estimated at $546 \mathrm{~nm}$. The measured percent scavenging activity was compared with ascorbic acid used as standard.

\section{Hydroxyl ion radical scavenging assay}

Different concentrations of extracts (250-1500 $\mu \mathrm{g} /$ $\mathrm{ml}$ ) were placed in a test tube and evaporated to dryness. To each tube, iron-EDTA solution (0.13\% ferrous ammonium sulfate and 0.26\% EDTA; $1 \mathrm{ml}$ ), EDTA (0.5 ml; 0.018\%), DMSO $(0.85 \%, v / v$ in $0.1 \mathrm{M}$ phosphate buffer, $\mathrm{pH} 7.4)$ and ascorbic acid ( $0.5 \mathrm{ml}$; $0.22 \%)$ were added. The tubes were capped tightly and heated in a water bath at $80-90^{\circ} \mathrm{C}$ for $15 \mathrm{~min}$. The reaction was terminated by adding $1 \mathrm{ml}$ 
of ice-cold TCA $(17.5 \% \mathrm{w} / \mathrm{v})$. Three $\mathrm{ml}$ of Nash reagent (75.0 g ammonium acetate, $3 \mathrm{ml}$ glacial acetic acid and $2 \mathrm{ml}$ acetyl acetone were mixed and water was added to a total volume of $1 \mathrm{l}$ ) was added to each tube and left at room temperature for 15 min for color development. The intensity of the yellow color formed was measured at $412 \mathrm{~nm}$ against a blank of the reagent. Percentage inhibition was determined by comparing the results of the test extracts with BHA.

\section{Superoxide anion radical scavenging assay}

For measurement of anion radical scavenging potential, reaction mixture included nitro blue tetrazolium $(\mathrm{pH}=7.4$; $1 \mathrm{ml} ; 156 \mathrm{mM})$, NADH solution ( $\mathrm{pH}=7.4 ; 1 \mathrm{ml} 468 \mathrm{mM})$, and different concentrations of extracts (250-1500 $\mu \mathrm{g}$ / $\mathrm{ml}$ ). To initiate the reaction, $100 \mathrm{ml}$ of $60 \mathrm{mM}$ phenazine methosulfate $(\mathrm{pH}=7.4)$ was added to the mixture and incubated at $25^{\circ} \mathrm{C}$ for $5 \mathrm{~min}$. Absorbance was measured at $560 \mathrm{~nm}$ against blank and was compared with standards as described previously. The following formula was used to calculate $\%$ inhibition of superoxide anion generation:

$$
\text { Inhibition }(\%)=\frac{A_{0}-A_{1}}{A_{0}} \times 100 \text {, }
$$

where $A_{0}=$ absorbance of the control and $A_{1}=a b$ sorbance of the extract. Synthetic antioxidant (BHA) and natural antioxidant ( $\alpha$-tocopherol), at 200 ppm, were used for comparison.

\section{Preparation of cosmetic emulsions}

For the formulation oily phase composed of paraffin oil (14\%) and emulsifier Abil-EM90 ${ }^{\circ}$ (2.5\%) were heated together up to $75 \pm 1^{\circ} \mathrm{C}$. At the same time distilled water (quantity sufficient to make $100 \%$ ) was heated at the same temperature and then 4\% Tamarindus indica seeds extract was added. After that, aqueous phase was added to the oil phase drop by drop. Stirring was continued at 2000 rpm by the mechanical mixer for about 15 min until complete aqueous phase was added, 2 to 3 drops of rose oil were added during continuous stirring to give good fragrance to the formulation. After the complete addition of the aqueous phase, the speed of the mixer was reduced to $1000 \mathrm{rpm}$ for homogenization, for a period of $5 \mathrm{~min}$, and then the speed of the mixer was further reduced to 500 rpm for 5 min for complete homogenization; until the emulsion cooled to room temperature. Base was also prepared by the same method and with same ingredients but without the Tamarindus indica seeds extract.

\section{Study design and subjects}

The study was designed as a home-use, single-blind, randomized side of cheeks and placebo controlled trial. The study was conducted in accordance with the ethics principles of the Helsinki Declaration. The protocol was approved by the Board of Advanced Studies and Re- search (BASR), and its Ethical Committee for in vivo Studies (Reference No. 4510/Acad.). Written informed consent was obtained from all participating volunteers.

Eleven male volunteers were selected whose ages were between 25 and 35 years. Inclusion/exclusion criteria were followed as per standards. Each volunteer was provided with the two emulsions. One emulsion was the base (B) and other was the active formulation (F) containing Tamarindus indica seeds extract. The volunteers were advised to use an amount which can be absorbed easily on the skin. Each volunteer applied emulsion for a period of 12 weeks. Values for different parameters were taken in controlled room temperature $25 \pm 1^{\circ} \mathrm{C}$ and $45 \pm 2 \%$ relative humidity. Every volunteer was instructed to come for measurement on the $2^{\text {th }}, 4^{\text {th }}, 6^{\text {th }}, 8^{\text {th }}, 10^{\text {th }}$ and $12^{\text {th }}$ week. Values for each parameter were measured three times $(n=3)$ and average of the values has been noted.

\section{Mathematical analysis}

The percentage changes for the individual values of different parameters, taken every week of volunteers were calculated by the following formula: Percentage change $=[(A-B) / B] \times 100$ where: $A=$ individual value of any parameter of the $2^{\text {nd }}, 4^{\text {th }}, 6^{\text {th }}, 8^{\text {th }}, 10^{\text {th }}$ and $12^{\text {th }}$ week, $\mathrm{B}=0 \mathrm{~h}$ value of that parameter.

\section{Statistical analysis}

The evaluation of data was done by using SPSS version 19.0 according to two-way ANOVA for variation between different time intervals defining a $5 \%$ level of significance and paired samples $t$-test for variation between the two preparations. Standard error of means (SEM) was calculated for every mean value.

\section{Results and discussion}

\section{Anti-oxidant activity}

$\mathrm{DPPH}$ radical scavenging activity indicated that Tamarindus indica seeds extract has marked antioxidant activity. Nitric oxide radical scavenging activity also indicated significant potential of the extract to quench NO radicals. Superoxide anion and hydroxyl ion scavenging activity similarly also indicated sufficient antioxidant potential of Tamarindus indica seeds extract. Antioxidant activity of Tamarindus indica seeds extract is presented in a table (Table 1).

The considerable antioxidant activity of Tamarindus indica $L$. seeds extract is due to the presence of 2-hydroxy-3',4'-dihydroxyacetophenone, methyl 3,4-dihydroxybenzoate, 3,4-dihydroxyphenylacetate and (-)-epicatechin, in addition to oligomeric proanthrocyanidines (OPCs) [6, 7]. Procyanidins, in addition to scavenging free radicals, strongly and non-competitively inhibit xanthine oxidase activity, the enzyme which triggers the oxy-radical cascade. Other studies have confirmed proanthocy- 
Table 1. In vitro antioxidant activity $\left(\mathrm{IC}_{50} \mu \mathrm{g} / \mathrm{ml}\right)$ of Tamarindus indica seeds extract

\begin{tabular}{lcc}
\hline Assay & $\begin{array}{c}\text { Tamarindus } \\
\text { indica }\end{array}$ & $\begin{array}{c}\text { Ascorbic } \\
\text { acid }\end{array}$ \\
\hline $\begin{array}{l}\text { Nitric oxide radical inhibition } \\
\text { activity }\end{array}$ & $118.94 \pm 1.14$ & $65.05 \pm 1.02$ \\
\hline DPPH radical scavenging assay & $99.08 \pm 1.30$ & $32.35 \pm 2.028$ \\
\hline $\begin{array}{l}\text { Hydroxyl radical scavenging } \\
\text { assay }\end{array}$ & $89.47 \pm 0.95$ & $75.92 \pm 1.23$ \\
\hline $\begin{array}{l}\text { Superoxide radical scavenging } \\
\text { assay }\end{array}$ & $105.27 \pm 1.38$ & $48.22 \pm 1.15$ \\
\hline
\end{tabular}

Data are expressed as means \pm standard deviations $(n=3)$.

anidins that provide protection against free radicals in in vitro free radical scavenging assay and this effect was better than vitamins $\mathrm{C}$ and $\mathrm{E}$ [8].

\section{Skin elasticity}

The capability of human skin to distend and then returning back to its normal state afterward is called elasticity. A loss of skin elasticity is a natural part of aging termed elastosis [9]. Photo aging is induced by constant exposure to ultraviolet (UV) irradiation and damages human skin. Antioxidants tender protection to cell membranes as they neutralize free radicals and noxious oxygen molecules and prevent oxidative stress to the tissues of the body. They supply elasticity to the skin by actively countering free radical attacks [10-12].

In this study, the skin elasticity of the volunteers was measured at specified intervals for 12 weeks. The irregular pattern in the values of skin elasticity was observed by the base but there was a regular increase in skin elasticity after the application of formulation throughout the study duration as shown in Figure 1. The ANOVA test proved that the variations in values of skin elasticity by formulation was significant whereas with base it was insignificant ( $p \leq 0.05)$ with respect to time. By applying paired sample $t$-test it was obvious that significant differ- ences in skin elasticity values were noticed after application of the formulation throughout the study duration.

The improvement in skin elasticity after application of the formulation is due to the presence of strong antioxidants, proanthocyanidins (PAs) in tamarind seed extract which increase the collagen synthesis and accelerate the conversion of soluble collagen to insoluble collagen during development and decrease the rate of enzymatic degradation of collagen matrices [13].

The PAs can also inhibit the catabolism of soluble collagen in animal studies [14].

\section{Skin moisture contents}

Aging of the skin is a complex phenomenon that happens as a result of numerous factors. To stop aging, it is necessary to shield the skin from damage. Protection should be taken against environmental factors [15]. The moisture of the skin should be restored by preventing the loss of moisture in the stratum corneum layer. The increase in the moisture of stratum corneum supplies a smoother and more even look [16]. In this study skin moisture content of the volunteers was measured by state-of-the-art Corneometer at specified intervals for 12 weeks. The study showed a regular increase in the moisture content in case of formulation but the effect of the base was not regular (Figure 2). The ANOVA test showed that the effect of formulation was significant $(p \leq 0.05)$ whereas the base has insignificant $(p>0.05)$ effects on the skin moisture content with respect to time. Moreover, the paired sample $t$ test proved that there had been significant differences in moisture values at all specified intervals.

It is reported that antioxidants have the ability to stimulate dermal fibroblasts for the synthesis of collagen, as the collagen intensity is increased, the hydration level also enhanced [17]. Oligomeric proanthrocyanidines (OPC) are potent antioxidants; present in Tamarindus indica seeds extract produced a significant increase in the hydration level of the stratum corneum $[18,19]$.

Mineral oils are primarily composed of lipids, filling the spaces between cells of stratum corneum. Emollients

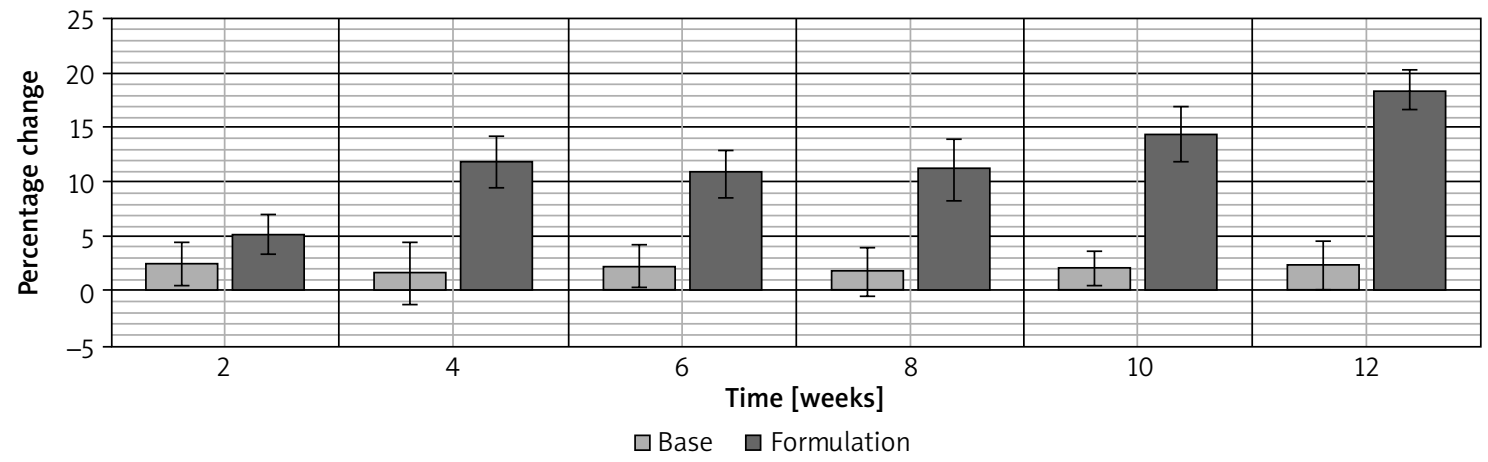

Figure 1. Percentage changes in skin elasticity after application of the base and formulation 


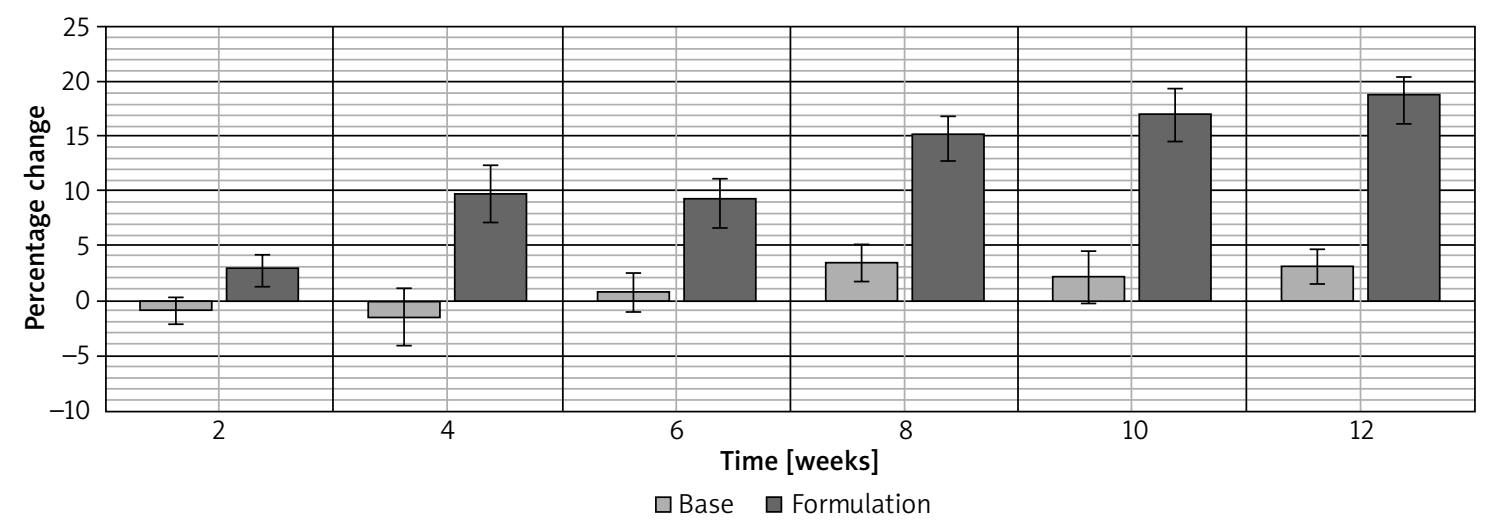

Figure 2. Percentage changes in skin moisture contents after application of the base and formulation

form a film of lipid on skin surface which restores the barrier function of the skin. Water holding capacity of the stratum corneum is enhanced by this film leading to reduced epidermal water loss $[20,21]$.

\section{Surface evaluation of living skin (SELS)}

The values of different SELS parameters SEr, SESC, SEsm and SEW measured by Visioscan ${ }^{\oplus}$ VC 98/software SELS 2000 before application of emulsions ( $0 \mathrm{~h}$ readings) and at the $1^{\text {st }}, 2^{\text {nd }}$ and $3^{\text {rd }}$ month of the study period. SEr is the roughness parameter which depicts the asperity of the skin and calculates the gray levels above the threshold in comparison with the whole image. It calculates the proportion of dark pixels. SEsm is the index of smoothness and is calculated from mean width and depth of wrinkles. SEsc is the index representing scaliness of skin which shows the level of dryness of the stratum corneum i.e. state of dehydration of the skin. It is the number of pixels where the gray level is higher than the threshold of SEsc. SEw identifies aging including wrinkles and is calculated from the proportion of horizontal and vertical wrinkles [22]. SELS parameters SEw, SEsc, SEsm and SEr before the application of emulsions and then at the $1^{\text {st }}, 2^{\text {nd }}$ and $3^{\text {rd }}$ months of the study duration are given in Table 2 and Figure 3 depicts images taken before and after three months of the application of formulation. In this study it was found that the base produced statistically insignificant $(p>0.05)$ effects on the roughness parameter of skin and the formulation produced significant $(p \leq 0.05)$ effects when ANOVA two way analysis was performed. When paired sample $t$-test was applied, significant effects were observed for active formulation. A gradual decrease in the values of roughness, scaliness, smoothness and wrinkles was observed for the formulation. This is also obvious from Figures $4 \mathrm{~A}$ and $\mathrm{B}$, where the visible differences can be seen between the peaks of the 3D image. The formulation showed a decrease in mean values of skin smoothness in contrast to skin roughness which indicates that the formulation possess anti-aging properties. Lower values of skin scaliness showed that the skin got hydrated with the passage of time during treatment. It was also supported by the values obtained by Corneometer ${ }^{\circledR}$ MPA 5 for skin hydration. The smaller SESC value corresponds to higher skin moisture as treatment with moisturizing or anti-aging formulations let the values for SEsc go down. Overexposure to the sunlight results in many of the skin changes associated with aging characterized by fine wrinkling and skin laxity. Collagen is responsible for imparting the skin pliability and elasticity. Decreased levels of collagen occur in both chronologic and photoaging. As collagen fibers serve as the primary structural support of the skin, it fol-

Table 2. SELS parameter values (mean \pm SD)

\begin{tabular}{|c|c|c|c|c|c|}
\hline Parameter & Emulsion & 0 hour & $1^{\text {st }}$ month & $2^{\text {nd }}$ month & $3^{\text {rd }}$ month \\
\hline \multirow[t]{2}{*}{ SEr } & Base & $4.02 \pm 0.054$ & $4.05 \pm 0.09$ & $4.01 \pm 0.080$ & $4.06 \pm 0.075$ \\
\hline & Formulation & $4.03 \pm 0.082$ & $3.98 \pm 0.063$ & $3.92 \pm 0.073$ & $3.82 \pm 0.081$ \\
\hline \multirow[t]{2}{*}{ SESC } & Base & $1.73 \pm 0.040$ & $1.73 \pm 0.048$ & $1.72 \pm 0.053$ & $1.72 \pm 0.056$ \\
\hline & Formulation & $1.71 \pm 0.038$ & $1.68 \pm 0.051$ & $1.63 \pm 0.053$ & $1.60 \pm 0.045$ \\
\hline \multirow[t]{2}{*}{ SEsm } & Base & $107.99 \pm 4.81$ & $109.98 \pm 4.80$ & $108.64 \pm 3.78$ & $107.82 \pm 2.82$ \\
\hline & Formulation & $101.01 \pm 4.65$ & $97.51 \pm 4.80$ & $95.12 \pm 2.33$ & $89.02 \pm 2.12$ \\
\hline \multirow[t]{2}{*}{ Sew } & Base & $70.63 \pm 1.42$ & $71.72 \pm 1.34$ & $70.84 \pm 1.34$ & $71.80 \pm 1.00$ \\
\hline & Formulation & $65.73 \pm 1.42$ & $63.73 \pm 1.33$ & $61.82 \pm 1.35$ & $58.31 \pm 1.34$ \\
\hline
\end{tabular}

SEr - skin roughness, SEw - skin wrinkles, SEsm - skin smoothness, SEsC - skin scaliness. 

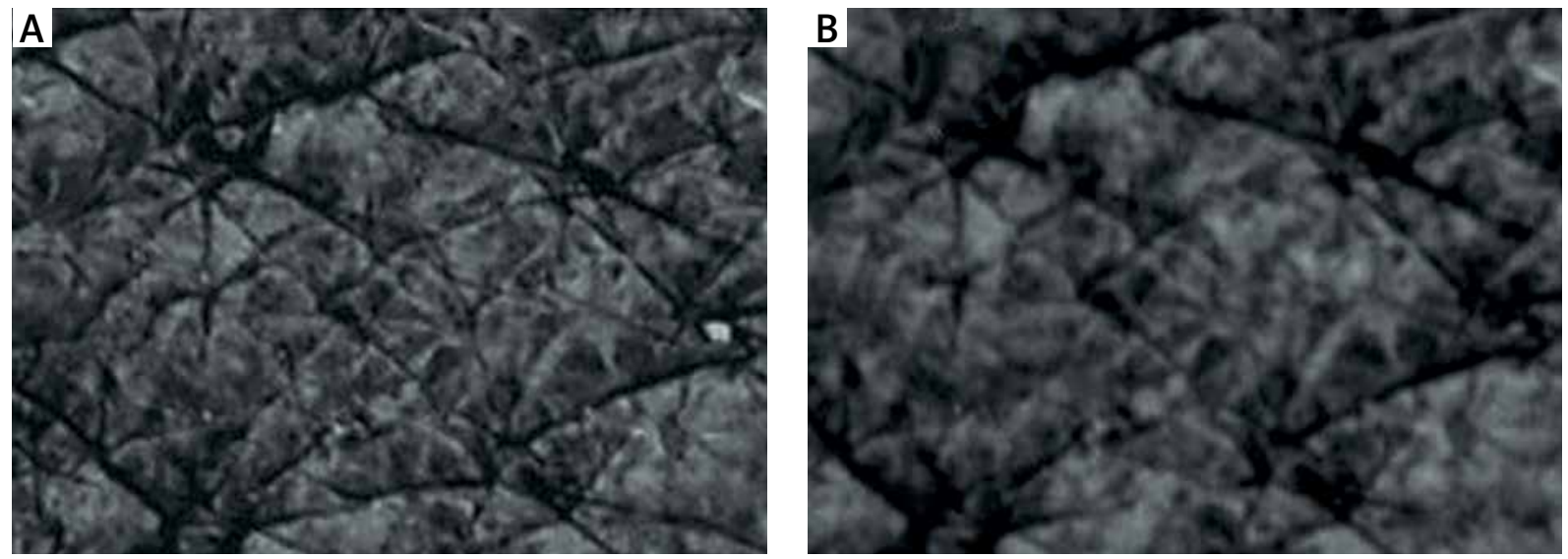

Figure 3. An image of skin taken by Visioscan ${ }^{\circledR}$ before $(\mathbf{A})$ and after 3 months (B) of tamarind formulation application
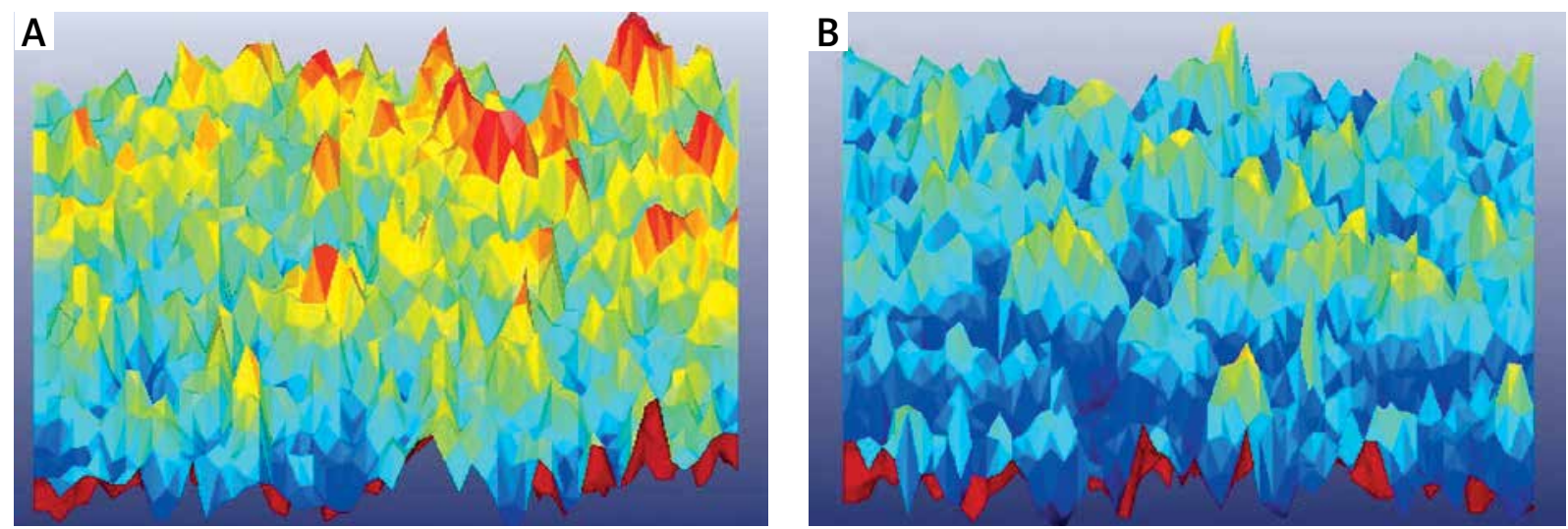

Figure 4. 3D image of skin taken by Visioscan ${ }^{\circledR}(\mathbf{A})$ before application of tamarind formulation (B) after 3 months of application of Tamarind formulation

lows logically that a reduction in skin collagen levels would be associated with the formation of skin wrinkles [23].

Lower values for the parameter SEw indicate that there were less wrinkles present on the skin which indicates that the formulation reduced the fine wrinkles and improved the appearance of the skin.

The improvement in skin surface parameters can be attributed to the phenolic compounds and flavonoids present in tamarind seeds extract which include 2-hydroxy-3',4'dihydroxyacetophenone, metdihydroxybenzoate, 3,4-dihydroxyphenylacetate and (-)-epicatechin, in addition to OPC. There is much supporting evidence present that phenolic compounds contribute towards the antiaging mechanism by providing an antioxidative benefit to the skin [24].

\section{Conclusions}

This study illustrates that tamarind (Tamarindus indica) seeds extract containing botanical antioxidants exhibits potent antiaging effects when topically applied. A stable cosmetic W/O emulsion loaded with Tamarindus indica seeds extract can be formulated. The formulation was noticed to increase the stratum corneum water contents. The increase in stratum corneum water contents provides a smoother look and protects the skin from aging. The decline in SELS parameters represents that antioxidants present in the formulation containing Tamarindus indica seeds extract possess anti-wrinkle effects. Furthermore, the botanical antioxidants in formulation displayed no deleterious effects so this formulation is economical and safe for usage in the management of facial wrinkles.

\section{Acknowledgments}

The authors are thankful to the higher education commission of Pakistan (HEC) for financial support.

\section{Conflict of interest}

The authors declare no conflict of interest.

\section{References}

1. Gilchrest BA. Skin aging and photoaging: an overview. J Am Acad Dermatol 1989; 21: 610-3. 
2. Masaki H. Role of antioxidants in the skin: anti-aging effects. J Dermatol Sci 2010; 58: 85-90.

3. Afaq F, Mukhtar H. Botanical antioxidants in the prevention of photocarcinogenesis and photoaging. Exp Dermatol 2006; 15: 678-84.

4. Nielloud F. Pharmaceutical Emulsions and Suspensions: Revised and Expanded. CRC Press 2000.

5. Luzia DM, Jorge N. Antioxidant activity, fatty acid profile and tocopherols of Tamarindus indica L. seeds. Food Sci Technol 2011; 31: 497-501.

6. Ditre C, Wu J, Baumann LS, Rigel D. Innovations in natural antioxidants and their role in dermatology. Cutis 2008; 82 (Suppl 6): 2-16.

7. Siddhuraju P. Antioxidant activity of polyphenolic compounds extracted from defatted raw and dry heated Tamarindus indica seed coat. Sci Technol 2007; 40: 982-90.

8. Hatano T, Miyatake H, Natsume M, et al. Proanthocyanidin glycosides and related polyphenols from cacao liquor and their antioxidant effects. Phytochemistry 2002; 59: 749-58.

9. Cua A, Wilhelm KP, Maibach H. Elastic properties of human skin: relation to age, sex, and anatomical region. Arch Dermatol Res 1990; 282: 283-8.

10. Yokozawa T, Lee YA, Cho EJ, et al. Anti-aging effects of oligomeric proanthocyanidins isolated from persimmon fruits. Drug Discover Ther 2011; 5: 109-18.

11. Cronin H, Draelos ZD. Top 10 botanical ingredients in 2010 anti aging creams. J Cosmet Dermatol 2010; 9: 218-25.

12. Goihman-Yahr M. Skin aging and photoaging: an outlook. Clin Dermatol 1996; 14: 153-60.

13. Egawa M, Oguri M, Kuwahara T, Takahashi M. Effect of exposure of human skin to a dry environment. Skin Res Technol 2002; 8: 212-8.

14. Khan BA, Akhtar N, Braga VA. Anti-aging effects of Hippophae rhamnoides emulsion on human skin. Trop J Pharmaceut Res 2013; 11: 955-62.

15. Skerget M, Kotnik P, Hadolin M, et al. Phenols, proanthocyanidins, flavones and flavonols in some plant materials and their antioxidant activities. Food Chem 2005; 89: 191-8.

16. Sato T, Sakamoto W, Odanaka W, et al. Clinical effects of dietary hyaluronic acid on dry, rough skin. Aesth Dermatol 2002; 12: 109-20.

17. Agero A, Verallo-Rowell VM. A randomized double-blind controlled trial comparing extra virgin coconut oil with mineral oil as a moisturizer for mild to moderate xerosis. Dermatitis 2004; 15: 109-16.

18. Pandey KB, Rizvi SI. Plant polyphenols as dietary antioxidants in human health and disease. Oxid Med Cell Longev 2009; 2: 270-8.

19. Khan BA, Akhtar N, Waseem K, et al. Visio Scan ${ }^{\circledR}$ VC98, Corneometer MPA 5 and Tewameter MPA 5. Afr J Pharm Pharmacol 2012; 6: 225-7.

20. Farage MA, Miller KW, Berardesca E, Maibach HI. Clinical implications of aging skin. Am J Clin Dermatol 2009; 10: 73-86.

21. Farage MA, Miller KW, Maibach HI. Degenerative changes in aging skin. Textbook of Aging Skin. Springer; 2010; 25-35.

22. Sudjaroen $\mathrm{Y}$, Haubner R, Würtele G, et al. Isolation and structure elucidation of phenolic antioxidants from Tamarind (Tamarindus indica L.) seeds and pericarp. Food Chem Toxicol 2005; 43: 1673-82.

23. Rice-Evans CA, Miller NJ, Paganga G. Structure-antioxidant activity relationships of flavonoids and phenolic acids. Free Rad Biol Med 1996; 20: 933-56.

24. Fine AM. Oligomeric proanthocyanidin complexes: history, structure, and phyto pharmaceutical applications. J Clin Ther 2000; 5: 144-51. 